\title{
Mechanical ventilation in idiopathic pulmonary fibrosis: a nationwide analysis of ventilator use, outcomes, and resource burden
}

\author{
Joshua J. Mooney ${ }^{1}$, Karina Raimundo ${ }^{2^{*}}$, Eunice Chang ${ }^{3}$ and Michael S. Broder ${ }^{3}$
}

\begin{abstract}
Background: Idiopathic pulmonary fibrosis (IPF) is associated with increased risk of respiratory-related hospitalizations. Studies suggest mechanical ventilation (MV) use in IPF does not improve outcomes and guidelines recommend against its general use. Our objective was to investigate MV use and association with cost and mortality in IPF.

Methods: This retrospective study, using a nationwide sample, included claims with IPF (ICD-9-CM: 516.3) in 2009-2011 and principal respiratory disease diagnosis (ICD-9-CM: 460-519); excluding lung transplant. Regression models were used to determine predictors of MV and association with cost, LOS, and mortality. Domain analysis was used to account for use of subpopulation. Costs were adjusted to 2011. Data on patient severity not available.

Results: Twenty two thousand three hundred fifty non-transplant IPF patients were admitted with principal respiratory disease diagnosis: Mean age 70.0 (SD 13.9), 49.1\% female, mean LOS 7.4 (SD 8.2). MV was used in 11.4\% of patients with a non-significant decline over time. In regression models, MV was associated with an increased stay of 9.78 days (95\% Cl 8.38-11.18) and increased cost of $\$ 36,583$ (95\% Cl $\$ 32,021-41,147)$. MV users had significantly increased mortality (OR 15.55, 95\% Cl 12.13-19.95) versus nonusers.

Conclusions: Mechanical ventilation use has not significantly changed over time and is mostly used in younger patients and those admitted for non-IPF respiratory conditions. MV was associated with a 4-fold admission cost increase ( $\$ 49,924$ versus $\$ 11,742$ ) and a 7 -fold mortality increase (56\% versus $7.5 \%)$, although patients who receive MV may differ from those who do not. Advances in treatment and decision aids are needed to improve outcomes in IPF.
\end{abstract}

Keywords: Mechanical ventilation, Mortality, Outcomes, Cost of illness, Idiopathic pulmonary fibrosis, Noninvasive ventilation

\section{Background}

Idiopathic pulmonary fibrosis (IPF), a form of interstitial pneumonia, affects $0.5 \%$ of US adults over age 65 [1]. The disease is characterized by progressive lung fibrosis [2] and unpredictable episodes of disease worsening, which may lead to hospitalization and frequently death [3-5]. The median survival from diagnosis is $3-5$ years [6]. Although two pharmacologic treatments that slow physiologic decline are now available $[7,8]$, limited options remain for IPF patients hospitalized with respiratory-related symptoms or failure.

\footnotetext{
* Correspondence: raimundo.karina@gene.com

${ }^{2}$ Genentech, Inc, South San Francisco, CA, USA

Full list of author information is available at the end of the article
}

Management of respiratory failure in IPF is challenging as patients can develop acute disease episodes that necessitate ventilator support. In select IPF patients, ventilator support can be used as a bridge to lung transplant $[9,10]$ or could allow for treatment of reversible non-IPF causes of respiratory failure. However, overall outcomes of IPF patients who require non-invasive ventilation or mechanical ventilation (MV) are poor [11-16]. A systematic review [17] summarizing 9 single-center studies reported an $87 \%$ in-hospital mortality rate for IPF patients who received MV. Given this evidence, IPF treatment guidelines recommend the majority of IPF patients with respiratory failure not receive MV, and when used should occur 
after assessing patient-specific goals of care or lung transplant candidacy [10].

While studies have repeatedly demonstrated high mortality with MV, the nationwide pattern of its use in IPF patients has not been well characterized. In this study, we investigated US trends in the use of non-invasive ventilation and MV for IPF, predictors of use, and association with hospital cost, length of stay (LOS), and mortality. We also examined whether MV had a direct effect on mortality, or whether the effect was entirely mediated by the patients' underlying disease and comorbid conditions.

\section{Methods}

\section{Design and data sources}

We conducted a retrospective cohort study using the Nationwide Inpatient Sample (NIS), the largest publicly available US inpatient database that includes individuals covered by Medicare, Medicaid, or private insurance, as well as the uninsured. Data elements include diagnoses, procedures, demographics, hospital characteristics, payment source, charges, discharge status, LOS, and severity measures [18]. The study used de-identified data and was exempt from institutional review board review.

\section{Patient Population}

We included all hospitalizations from 2009-2011 with claims for IPF (International Classification of Diseases, 9th Revision, Clinical Modification [ICD-9-CM] code 516.3) and a principal diagnosis of respiratory disease (ICD-9-CM: 460-519). Hospital discharge records may contain multiple diagnoses, with the primary cause for admission listed as "principal." A hospitalization for a patient with IPF admitted with pneumonia as the principal diagnosis would have been included in our study, as pneumonia is a respiratory disease, as would a hospitalization with a principal diagnosis of IPF (also a respiratory disease). An admission with a principal diagnosis of hip fracture would not be included, even if IPF was listed as a secondary diagnosis. Included patients had $\geq 1$ inpatient claim with IPF as a discharge diagnosis between 2009-2011. We excluded lung transplant admissions (ICD-9-CM: 33.5×, 33.6).

\section{Variables}

Outcome variables of interest were non-invasive (ICD-9CM: 93.90) and MV (ICD-9-CM: 96.7x) use, hospital LOS, total inpatient costs, and in-hospital mortality. Other study variables include demographics, primary payer type, hospital characteristics, and all patient refined diagnosis-related group (APR-DRG) severity of illness. APR-DRG assigns patients to severity and mortality subclasses using comorbidities, age, procedures, and principal diagnosis [19]. We looked for evidence of concomitant acute and chronic pulmonary conditions, including chronic obstructive pulmonary disease (COPD), bacterial pneumonia, and lung cancer. Cardiovascular conditions were identified, including ischemic heart disease, myocardial infarction (MI), congestive heart failure, and pulmonary hypertension. The number of chronic conditions for each patient, calculated using the Chronic Condition Indicator, was reported. This indicator uses 5 digit ICD-9-CM codes to categorize conditions as chronic or not chronic [20]. Admissions were characterized as elective, emergency, urgent, or other non-elective. Discharge disposition was reported as routine, transfer to short-term hospital, transfer to other facilities, home health care, died in hospital, or unknown.

\section{Statistical analysis}

Variables were weighted to represent national estimates and rounded to the nearest integer. NIS reports only charges, so cost-to-charge ratios were used to estimate costs. These ratios are constructed using costs and charge information from hospital reports to CMS. Hospitalspecific ratios were used if available; otherwise a weighted group average was used. Costs were adjusted to 2011 US\$ using the medical care component of the consumer price index [21]. For categorical variables, Rao-Scott chi-square goodness-of-fit tests adjusting for sampling design were used, relevant $\mathrm{p}$-values reported. We calculated variance using domain analysis to account for subpopulations. Linear regression models were used for LOS and cost, logistic regression models for MV and mortality. Models were adjusted for age, gender, race, principal diagnosis of IPF, lung cancer, selected cardiovascular conditions, hospital region, hospital teaching status, and MV use, as appropriate. Adjusted mean LOS and hospital cost, and adjusted inpatient mortality rate (and 95\% confidence intervals) were reported for MV users and nonusers.

Patients with certain characteristics may have a higher risk of inpatient mortality and MV use. To investigate whether MV use was a mediator between clinical characteristics and mortality (rather than directly related), we followed the approach described by Baron and Kenny [22]. We conducted additional regression models to examine the association of clinical conditions/characteristics (the causal variables) on both the MV use (the mediator) and mortality (the outcome variable). Model results were compared to determine whether mediation effects were identifiable. Data transformations and statistical analyses were performed using $\mathrm{SAS}^{\bullet}$ version 9.4.

\section{Results}

From 2009-2011 42,924 IPF patients were admitted to US short-stay hospitals; 23,739 admissions had a principal diagnosis of respiratory disease. The remainder of admissions for these IPF patients were for non-respiratory conditions. After excluding 1,379 lung transplant admissions and 10 with missing age, final sample size was 22,350: 7,346 in 2009, 6,643 in 2010, and 8,362 in 2011. MV was 
used in $11.4 \%(2,546)$ of admissions: $12.1 \%$ (887) in 2009 , $11.5 \%$ (764) in 2010, and $10.7 \%$ (894) in $2011(p=0.578)$. Non-invasive ventilation was used in $8.9 \%(1,995)$ of admissions: $7.9 \%$ (583) in 2009, 8.3\% (550) in 2010, and $10.3 \%$ (862) in $2011(p=0.112)$ (Fig. 1).

\section{Unadjusted analysis}

Mean age was $65.9(+/-0.62)$ for MV users and 70.5 $(+/-0.34)$ for nonusers $(p<0.001)$. Overall, $49.1 \%$ $(10,976)$ of patients were female: $40.2 \%(1,024)$ of $\mathrm{MV}$ users and $50.3 \%(9,953)$ of nonusers $(p<0.001)$. The majority $(64.4 \%, n=14,404)$ of patients were White, 9.4\% Hispanic, $7.6 \%$ Black, with no significant difference by MV use. The primary payer was Medicare for $58.9 \%$ of admissions at which MV was used, compared to $69.7 \%$ where it was not $(p<0.001)$. A principal diagnosis of IPF was present in $31.5 \%$ of admissions at which MV was used vs. $44.6 \%$ where it was not $(p<0.001)$ (Table 1$)$.

ICD-9-CM diagnoses of pneumonia $(49.2 \%$ vs. $37.1 \%, p<0.001)$ and $\mathrm{MI}(10.5 \%$ vs. $5.4 \%, p<0.001)$ were more common in patients requiring $\mathrm{MV}$, while COPD $(28.9 \%$ vs. $39.4 \%, p<0.001)$ was less common. As is the case for all diagnoses in this study, these conditions were not confirmed clinically. MV users had significantly fewer chronic conditions (4.2 vs. 4.3, $p<0.001$ ) (Table 2). Patients who used MV had longer hospital stays $(16.5$ days $[+/-0.73]$ vs. 6.2 $[+/-0.10], p<0.001)$, were more likely to have died in the hospital (55.3\% vs. $8.8 \%)$ and less likely to have a routine home discharge $(9.3 \%$ vs. $51.2 \%)(p<0.001)$. Costs $(\$ 49,924$ vs. $\$ 11,742, p<0.001)$ were higher in MV users compared to nonusers (Table 3 ).

\section{Adjusted analysis}

MV was associated with an adjusted LOS of 16.1 days (95\% CI: 15; 17.5) versus 6.3 days (95\% CI: 6; 6.5) for nonusers. The adjusted cost associated with MV was $\$ 48,772$ (95\% CI: 43,979; 53,565) versus \$11,861 (95\% CI: 11,292 ; 12,431) for nonusers. The adjusted in-hospital death rate for MV users and nonusers was 55.7\% (95\% CI: $50.3 ; 61.0)$ and $7.5 \%$ (95\% CI: 6.6; 8.4) (Table 4). Each year of increased age was associated with shorter LOS $(-0.03$; 95\% CI: $-0.06 ;-0.01)$ and lower cost $(\$-143 ; 95 \%$ CI: $-208 ;-78$ ) but greater in-hospital death (OR 1.02; 95\% CI: $1.01 ; 1.03)$. The use of non-invasive ventilation was associated with increased LOS (2.03 days; 95\% CI: 0.93; 3.14), cost $(\$ 5,119 ; 95 \%$ CI: 2,$000 ; 8,238)$ and death (OR 4.77; 95\% CI: 3.48; 6.55) (Fig. 2). A principal diagnosis of IPF was associated with increased cost $(\$ 1,731 ; 95 \% \mathrm{CI}$ : 636 ; 2,827 ) and death (OR 1.78; 95\% CI: 1.42; 2.24) but no change in LOS (Fig. 2).

To investigate the association among clinical conditions/characteristics, MV use, and inpatient mortality, we conducted two additional logistic regression models. In the model for risk of MV, we controlled for patient and hospital characteristics. In this model, decreasing age (OR 0.97, 95\% CI: 0.97; 0.98), female gender (OR 0.68, 95\% CI: 0.55; 0.85), Hispanic ethnicity (OR $0.66,95 \%$ CI: 0.45 ; 0.97 ) and principal diagnosis of IPF (OR 0.60, 95\% CI: 0.48; 0.76) were associated with a lower risk of MV. Cardiovascular conditions (OR 1.34, 95\% CI: $1.08 ; 1.65 ; p=0.007$ ), bacterial pneumonia (OR 1.55, 95\% CI: $1.27 ; 1.90 ; p<0.001)$, and teaching hospital admission (OR 1.58, 95\% CI 1.26; $1.98 ; p<0.001$ ) were associated with higher risk of MV. In the model for in-hospital death that excluded $\mathrm{MV}$ as a predictor, female gender was associated with a lower risk of death (OR 0.62, 95\% CI: 0.52; 0.74; $p<0.001$ ), whereas principal diagnosis of IPF (OR 1.26, 95\% CI: $1.03 ; 1.55 ; p=0.026)$, teaching hospital admission (OR $1.37,95 \%$ CI $1.11 ; 1.69 ; p=0.003)$, cardiovascular conditions (OR 1.26, 95\% CI: $1.04 ; 1.51 ; p=0.017)$, and

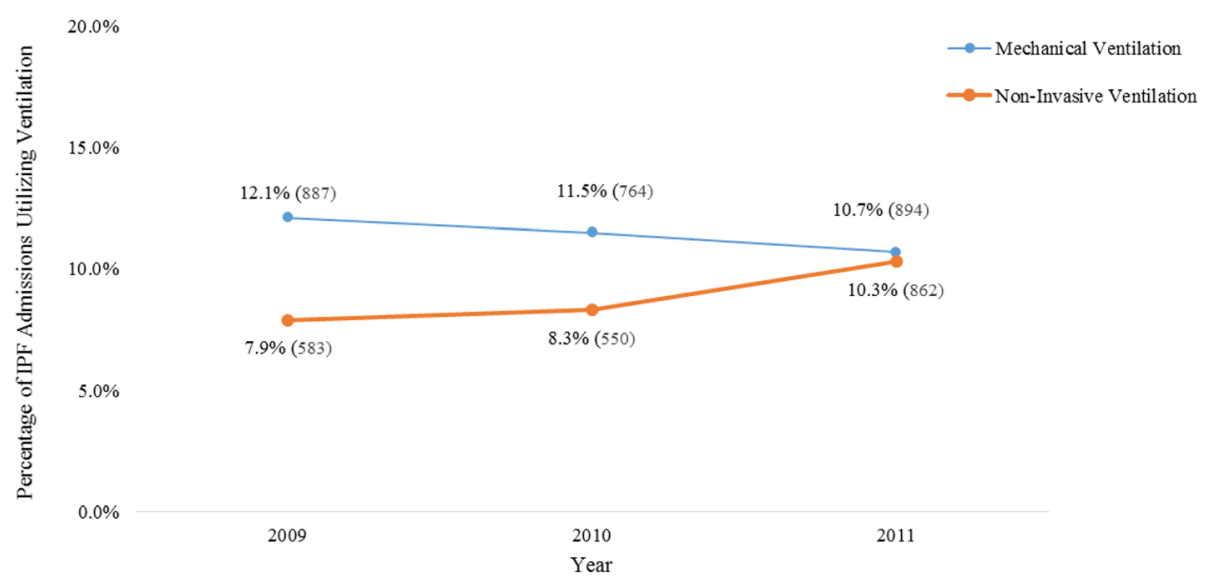

Fig. 1 Trend in Ventilation Use in IPF Hospitalizations. The proportion of IPF hospitalizations where mechanical ventilation was used declined each year, going from 12.1\% (887) in 2009, to $11.5 \%(764)$ in 2010, and 10.7\% (894) in 2011 ( $p=0.578)$. The use of non-invasive ventilation increased over the same period: $7.9 \%$ (583) in 2009, 8.3\% (550) in 2010, and 10.3\% (862) in 2011 ( $p=0.112$ ) 
Table 1 Patient Demographics, Hospital Characteristics, and Admission Type

\begin{tabular}{|c|c|c|c|c|}
\hline & Mean $(+/-S E) / r$ & & & $P$ \\
\hline & $\begin{array}{l}\mathrm{MV} \\
N=2,546\end{array}$ & $\begin{array}{l}\text { No MV } \\
N=19,805\end{array}$ & $\begin{array}{l}\text { All } \\
N=22,350\end{array}$ & \\
\hline Age & $65.9(+/-0.62)$ & $70.5(+/-0.34)$ & $70.0(+/-0.32)$ & $<.001$ \\
\hline Female & $1,024(40.2 \%)$ & $9,953(50.3 \%)$ & $10,976(49.1 \%)$ & $<.001$ \\
\hline Race & & & & 0.657 \\
\hline White & $1,639(64.4 \%)$ & $12,764(64.5 \%)$ & $14,404(64.4 \%)$ & \\
\hline Black & $224(8.8 \%)$ & $1,483(7.5 \%)$ & $1,707(7.6 \%)$ & \\
\hline Hispanic & $200(7.8 \%)$ & $1,910(9.6 \%)$ & $2,110(9.4 \%)$ & \\
\hline Other & $129(5.1 \%)$ & 999 (5.0\%) & $1,128(5.0 \%)$ & \\
\hline Missing & $353(13.9 \%)$ & $2,649(13.4 \%)$ & $3,002(13.4 \%)$ & \\
\hline Primary payer type & & & & $<.001$ \\
\hline Medicare & 1,499 (58.9\%) & $13,798(69.7 \%)$ & $15,297(68.4 \%)$ & \\
\hline Medicaid & $231(9.1 \%)$ & $1,300(6.6 \%)$ & $1,531(6.9 \%)$ & \\
\hline Private (including HMO) & $710(27.9 \%)$ & $3,880(19.6 \%)$ & $4,590(20.5 \%)$ & \\
\hline Self-pay & $41(1.6 \%)$ & $408(2.1 \%)$ & $448(2.0 \%)$ & \\
\hline Missing/No charge/Other & $65(2.5 \%)$ & $420(2.1 \%)$ & $484(2.2 \%)$ & \\
\hline Hospital region & & & & 0.845 \\
\hline Northeast & $433(17.0 \%)$ & $3,465(17.5 \%)$ & 3,897 (17.4\%) & \\
\hline Midwest & $607(23.8 \%)$ & $5,037(25.4 \%)$ & $5,644(25.3 \%)$ & \\
\hline South & $1,055(41.4 \%)$ & $8,114(41.0 \%)$ & $9,169(41.0 \%)$ & \\
\hline West & $452(17.8 \%)$ & $3,189(16.1 \%)$ & $3,641(16.3 \%)$ & \\
\hline Teaching hospital & $1,332(52.3 \%)$ & $8,354(42.2 \%)$ & $9,687(43.3 \%)$ & $<.001$ \\
\hline Bed size & & & & 0.022 \\
\hline Small & 229 (9.0\%) & 2,581 (13.0\%) & 2,811 (12.6\%) & \\
\hline Medium & 499 (19.6\%) & $4,309(21.8 \%)$ & 4,807 (21.5\%) & \\
\hline Large & $1,771(69.6 \%)$ & $12,676(64.0 \%)$ & $14,447(64.6 \%)$ & \\
\hline Missing & 47 (1.8\%) & $239(1.2 \%)$ & $286(1.3 \%)$ & \\
\hline Evidence of ED services $^{a}$ & $1,650(64.8 \%)$ & $13,262(67.0 \%)$ & $14,912(66.7 \%)$ & 0.363 \\
\hline Principal diagnosis of IPF & $802(31.5 \%)$ & $8,823(44.6 \%)$ & 9,626 (43.1\%) & $<.001$ \\
\hline Elective admission ${ }^{b}$ & $361(14.2 \%)$ & $3,152(15.9 \%)$ & $3,512(15.7 \%)$ & 0.307 \\
\hline
\end{tabular}

${ }^{2}$ Defined by NIS as having either an ED revenue code, charge, CPT procedure code, or admission source, or being on a state-defined ED record

${ }^{\mathrm{b}}$ Defined by NIS as admission other than emergency, urgent, newborn, delivery, trauma center, or other-non elective

bacterial pneumonia (OR 1.42. 95\% CI: 1.18; 1.71; $p<0.001$ ) were associated with increased risk (Table 5).

\section{Discussion}

Our study of IPF patients admitted to a nationwide sample of acute care hospitals found $11-12 \%$ of IPF patients admitted with a respiratory condition used MV, with no significant change from 2009-2011. Younger, male patients with fewer comorbidities and/or with a non-IPF principal diagnosis (e.g., pneumonia) were more likely to use MV. MV was associated with nearly 10-day longer hospital stays, $\$ 37,000$ higher cost, and a more than 7 -fold increase in mortality (56\% versus $7.5 \%$ ). Less than $10 \%$ of patients who used MV were discharged home routinely, compared to more than half of nonusers. Non-invasive ventilation was associated with increased LOS and cost, although to a lesser extent than MV.

The unchanging nationwide use of MV over time, despite IPF treatment guidelines conditionally recommending against MV use, reflects the limited options available to clinicians treating acute worsening of IPF and the difficulty of advance care planning in IPF. As acute worsening leading to respiratory failure can occur quickly and unexpectedly, MV can provide time to evaluate for possible treatable conditions, to assess patient preferences and/or to support gas-exchange while awaiting lung transplant. Lung transplantation remains the only curative and lifeprolonging option for select patients with advanced IPF and respiratory failure. Notably, IPF patients who received $\mathrm{MV}$ were younger with fewer chronic medical conditions, 
Table 2 Patient Clinical Characteristics and Treatment

\begin{tabular}{|c|c|c|c|c|}
\hline & Mean $(+/-$ SE) & & & $P$ \\
\hline & $\begin{array}{l}\mathrm{MV} \\
N=2,546\end{array}$ & $\begin{array}{l}\text { No MV } \\
N=19,805\end{array}$ & $\begin{array}{l}\text { All } \\
N=22,350\end{array}$ & \\
\hline No. of chronic conditions & $4.2(+/-0.06)$ & $4.3(+/-0.03)$ & $4.3(+/-0.03)$ & $<.001$ \\
\hline $\begin{array}{l}\text { Chronic obstructive pulmonary } \\
\text { disease }\end{array}$ & $736(28.9 \%)$ & 7,800 (39.4\%) & 8,535 (38.2\%) & $<.001$ \\
\hline Bacterial pneumonia & $1,252(49.2 \%)$ & 7,352 (37.1\%) & $8,604(38.5 \%)$ & $<.001$ \\
\hline Lung cancer & $59(2.3 \%)$ & $348(1.8 \%)$ & $407(1.8 \%)$ & 0.380 \\
\hline Cardiovascular conditions & $1,229(48.3 \%)$ & 8,835 (44.6\%) & $10,063(45.0 \%)$ & 0.137 \\
\hline Ischemic heart disease & 717 (28.2\%) & $5,622(28.4 \%)$ & 6,339 (28.4\%) & 0.913 \\
\hline Myocardial infarction & $267(10.5 \%)$ & $1,078(5.4 \%)$ & $1,345(6.0 \%)$ & $<.001$ \\
\hline Congestive heart failure & $793(31.1 \%)$ & 5,427 (27.4\%) & 6,219 (27.8\%) & 0.119 \\
\hline Pulmonary hypertension & $19(0.8 \%)$ & $65(0.3 \%)$ & $84(0.4 \%)$ & 0.146 \\
\hline APR-DRG severity of illness & & & & $<.001$ \\
\hline Minor loss of function & $5(0.2 \%)$ & $443(2.2 \%)$ & $447(2.0 \%)$ & \\
\hline Moderate loss of function & $16(0.6 \%)$ & $5,042(25.5 \%)$ & $5,058(22.6 \%)$ & \\
\hline Major loss of function & $341(13.4 \%)$ & $10,197(51.5 \%)$ & $10,538(47.1 \%)$ & \\
\hline Extreme loss of function & $2,184(85.8 \%)$ & $4,123(20.8 \%)$ & 6,307 (28.2\%) & \\
\hline
\end{tabular}

more often admitted at a teaching hospital, and more frequently coded with a non-IPF principal respiratory diagnosis (e.g., pneumonia). This suggests a nationwide preference for MV use in younger, somewhat healthier, IPF patients or in those with a clinical suspicion of a reversible condition. Possible explanations for this finding are that younger patients with less chronic comorbidity may be potential lung transplant candidates or clinicians may feel compelled to offer them a trial of ventilator support. We cannot ascertain from the data if patients were awaiting transplant or later transferred for transplant evaluation.
The overall economic and health care burden of IPF is well-recognized [23-27]. This study uniquely highlights the burden associated with MV use in IPF, while reinforcing with nationwide data the poor outcomes reported in prior smaller studies. Hospital cost was more than 4-fold greater and mortality 7-fold greater in IPF patients hospitalized with a respiratory problem requiring MV. While in-hospital mortality (55.3\%) was lower than previously reported, this underestimates mortality as a significant number of patients were transferred to short-term hospitals (6.9\%) or other facilities $(20.8 \%)$ where their final vital status is unknown. Only $16.4 \%$ of MV users were discharged home. The high

Table 3 Patient Discharge Status, LOS, and Total Costs

\begin{tabular}{|c|c|c|c|c|}
\hline & \multicolumn{3}{|l|}{ Mean (+/-SE)/no.(\%) } & \multirow{2}{*}{$\begin{array}{l}P \\
\text { Value }\end{array}$} \\
\hline & $\begin{array}{l}\text { MV Use } \\
N=2,546\end{array}$ & $\begin{array}{l}\text { No MV Use } \\
N=19,805\end{array}$ & $\begin{array}{l}\text { All } \\
N=22,350\end{array}$ & \\
\hline Discharge status & & & & $<.001$ \\
\hline Routine & $236(9.3 \%)$ & $10,131(51.2 \%)$ & $10,367(46.4 \%)$ & \\
\hline Transfer to short-term hospital & $175(6.9 \%)$ & $548(2.8 \%)$ & $724(3.2 \%)$ & \\
\hline Transfer to other facilities & $531(20.8 \%)$ & $3,353(16.9 \%)$ & $3,883(17.4 \%)$ & \\
\hline Home health care & $181(7.1 \%)$ & 3,937 (19.9\%) & $4,118(18.4 \%)$ & \\
\hline Died in hospital & $1,408(55.3 \%)$ & $1,738(8.8 \%)$ & $3,146(14.1 \%)$ & \\
\hline Other $^{a}$ & $15(0.6 \%)$ & $98(0.5 \%)$ & $112(0.5 \%)$ & \\
\hline Days of stay (among all IPF patients) & $16.5(+/-0.73)$ & $6.2(+/-0.10)$ & $7.4(+/-0.15)$ & $<.001$ \\
\hline Died in hospital & $1,408(55.3 \%)$ & $1,738(8.8 \%)$ & $3,146(14.1 \%)$ & $<.001$ \\
\hline Total inpatient costs (2011 US\$) & $\$ 49,924(+/-2,490)$ & $\$ 11,742(+/-390)$ & $\$ 16,042(+/-631)$ & $<.001$ \\
\hline
\end{tabular}

${ }^{a}$ Against medical advice, discharged alive, or destination unknown 
Table 4 Adjusted LOS, Inpatient Costs, and In-Hospital Death Rate ${ }^{a}$

\begin{tabular}{llll}
\hline IMV Use & Adjusted $^{\mathrm{a}}$ in-Hospital Death Rate $(95 \% \mathrm{Cl})$ & Adjusted $\mathrm{OR}^{\mathrm{O}}(95 \% \mathrm{Cl})$ & $P$ Value \\
\hline Yes & $55.7 \%(50.3-61.0)$ & $15.55(12.13-19.95)$ & $<.001$ \\
No & $7.5 \%(6.6-8.4)$ & ref &
\end{tabular}

Cl Confidence interval; OR Odds ratio

${ }^{a}$ Adjusted by age, gender, race, hospital region, teaching hospital, principal diagnosis of IPF, lung cancer, selected cardiovascular conditions (ischemic heart disease, myocardial infarction, and congestive heart failure), and non-invasive ventilatio4n use

mortality and economic burden associated with MV in IPF stresses the need to improve the quality of medical care for IPF patients, including advances in prevention, treatment, and patient-clinician shared decision-making. While recently approved pharmacologic therapies slow disease progression and may reduce acute exacerbations $[7,8,28]$, the course of IPF remains unpredictable. Therefore, early patient-centered discussions on treatment expectations, appropriate referrals for transplant and/or palliative care, and coordination of care across providers, remain integral to honoring patients' values while ensuring high value care. IPF-specific decision aids are needed to help guide patients.

Some conditions that lead to MV may themselves be associated with greater mortality, confounding the interpretation of our findings. We used a method similar to that of Baron and Kenny [22] to test whether MV simply
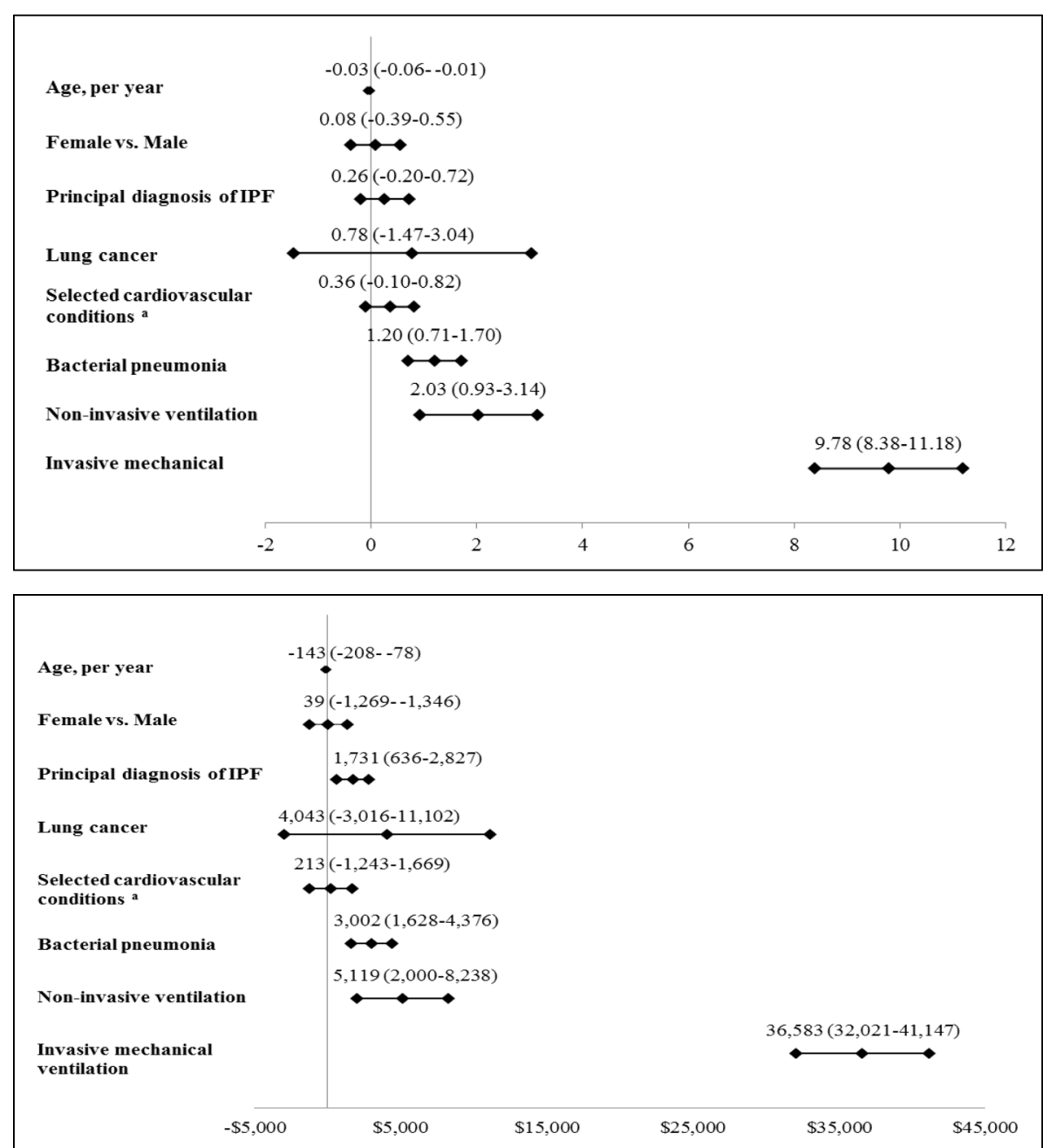

Fig. 2 Linear Regression Model for LOS and Costs. Age, bacterial pneumonia, and use of mechanical ventilation were statistically significantly ( $p<0.001$ ) associated with cost and LOS. Admission with a principal diagnosis of IPF was significantly associated with cost but not LOS. Use of mechanical ventilation had the largest effect on LOS and cost, with an increase of 9.78 days [95\% Cl: 8.38 - 11.18] and \$36,583 [32,021 - 41,147] respectively. Non-invasive ventilation was associated with an increase of 2.03 days $[0.93-3.14]$ in LOS and $\$ 5,119[2,000-8,238]$ in cost. Point estimates and $95 \% \mathrm{Cl}$ for LOS and cost are adjusted for all listed variables. Cl Confidence interval; ${ }^{a}$ Ischemic heart disease, myocardial infarction, and congestive heart failure 
Table $\mathbf{5}$ Logistic Regression Model Results for Risk of Mechanical Ventilation and Death

\begin{tabular}{|c|c|c|c|c|}
\hline \multirow[t]{3}{*}{ Parameter } & \multicolumn{4}{|c|}{ Logistic Regression Model } \\
\hline & \multicolumn{2}{|c|}{ Risk of In-Hospital Death } & \multicolumn{2}{|c|}{ Risk of Invasive Mechanical Ventilation } \\
\hline & OR $(95 \% \mathrm{Cl})$ & $P$ Value & OR $(95 \% \mathrm{Cl})$ & $P$ Value \\
\hline Age, per year & $1.00(1.00-1.01)$ & 0.562 & $0.97(0.97-0.98)$ & $<.001$ \\
\hline Female vs. Male & $0.62(0.52-0.74)$ & $<.001$ & $0.68(0.55-0.85)$ & $<.001$ \\
\hline \multicolumn{5}{|l|}{ Race } \\
\hline Black vs. White & $0.70(0.47-1.04)$ & 0.081 & $0.98(0.68-1.39)$ & 0.896 \\
\hline Hispanic vs. White & $0.80(0.56-1.15)$ & 0.235 & $0.66(0.45-0.97)$ & 0.036 \\
\hline Other vs. White & $1.05(0.68-1.62)$ & 0.836 & $0.86(0.52-1.42)$ & 0.547 \\
\hline Missing vs. White & $1.09(0.78-1.52)$ & 0.627 & $0.99(0.71-1.39)$ & 0.966 \\
\hline \multicolumn{5}{|l|}{ Hospital region } \\
\hline Northeast vs. West & $0.95(0.65-1.37)$ & 0.776 & $0.75(0.50-1.11)$ & 0.151 \\
\hline Midwest vs. West & $0.85(0.60-1.20)$ & 0.362 & $0.77(0.53-1.11)$ & 0.161 \\
\hline South vs. West & $0.92(0.68-1.25)$ & 0.605 & $0.82(0.59-1.12)$ & 0.207 \\
\hline Teaching hospital & $1.37(1.11-1.69)$ & 0.003 & $1.58(1.26-1.98)$ & $<.001$ \\
\hline Principal diagnosis of IPF & $1.26(1.03-1.55)$ & 0.026 & $0.60(0.48-0.76)$ & $<.001$ \\
\hline Lung cancer & $1.71(0.99-2.94)$ & 0.053 & $1.12(0.57-2.20)$ & 0.750 \\
\hline Selected cardiovascular conditions ${ }^{\text {a }}$ & $1.26(1.04-1.51)$ & 0.017 & $1.34(1.08-1.65)$ & 0.007 \\
\hline Bacterial pneumonia & $1.42(1.18-1.71)$ & $<.001$ & $1.55(1.27-1.90)$ & $<.001$ \\
\hline
\end{tabular}

OR Odds ratio, $\mathrm{Cl}$ Confidence interval

aschemic heart disease, myocardial infarction, and congestive heart failure

mediated the mortality effects of other variables. Our results suggest that this was not true of a principal IPF diagnosis, as it remains associated with mortality in the models both with and without MV use. Both cardiovascular conditions and bacterial pneumonia had statistically significant effects in the model that did not include MV, and smaller, not statistically significant, effects in the model that included it. This suggests the association between these characteristics and in-hospital death results, at least in part, from their association with $\mathrm{MV}$, which independently increases the risk of death. However, some residual confounding by indication likely still exists.

This study has limitations. First, there is debate on how to identify IPF patients using claims data. The ICD-9-CM code we used has been used before in several publications $[1,6,23,24]$, however a recent validation study (not in the NIS) found it had a positive predictive value of $30-60 \%$ [29]. While less than desirable, this positive predictive value is within the range reported in study of ICD-9-CM codes for 32 conditions (median $80.7 \%$, mean $77 \%$, range $23-100 \%$ ) [30]. Similarly, none of the conditions we identified (e.g., COPD) were confirmed clinically. Identification relied on ICD-9-CM codes, which are designed and used primarily for billing. Our study was further limited in that the NIS does not allow patients to be followed through subsequent outpatient care, repeat hospitalizations, or transfer to other facilities. We could not determine whether a subject died, received a transplant, or was discharged home after being transferred. The study relied on secondary data collected at discharge for administrative purposes, so no clinical information, including disease severity, was available. We could not determine whether medical conditions were present on admission or developed during hospitalization, nor could we determine the order in which diagnoses were made or treatments were given. Further, less severe comorbid conditions common to patients with IPF (e.g., obesity and gastroesophageal reflux) may be undercoded. Finally, as in prior studies [23, 24] we excluded transplantrelated expenditures. This exclusion allows for a close look at direct costs of IPF-related care, but underestimates the complete cost of IPF.

A strength of the study is the use of the Nationwide Inpatient Sample, which was designed to inform policy decisions regarding health and health care at national and regional levels. Previous evaluations of IPF MV use and cost have been limited to specific centers or populations (e.g., Medicare and select private insurers) and their findings may be less generalizable. The NIS includes patients with Medicare, Medicaid, and private insurance, as well as the uninsured, making this dataset the best way to produce estimates valid for the overall US population. 


\section{Conclusion}

In a nationwide sample of IPF patients, MV was used in $11-12 \%$ of those hospitalized due to a respiratory diagnosis with no significant change in its use over time. Mechanical ventilation was more frequent in younger male IPF patients, those admitted at teaching hospitals, and those with fewer chronic medical conditions or a non-IPF respiratory diagnosis. Its use was associated with a 4-fold increase in admission cost $(\$ 49,924$ compared to $\$ 11,742)$ and a 7 -fold increase in admission mortality (56\% compared to $7.5 \%)$. Further advances in IPF treatment and development of IPF-specific decision aids are needed to improve the resource burden, outcomes, and use of MV in IPF.

\section{Abbreviation}

APR-DRG: All patient refined diagnosis-related group; Cl: Confidence interval; COPD: Chronic obstructive pulmonary disease; CPT: Current procedural terminology; ED: Emergency department; ICD-9-CM: International classification of diseases, 9th revision, clinical modification; IPF: Idiopathic pulmonary fibrosis; LOS: Length of stay; MI: Myocardial infarction; MV: Mechanical ventilation; NIS: Nationwide inpatient sample; OR: Odds ratio; Revision: Clinical modification; SD: Standard deviation

\section{Acknowledgments}

Not applicable.

\section{Funding}

This study was funded by Genentech, Inc. The sponsor was involved in the study design, interpretation, and manuscript writing. K. Raimundo is an employee of Genentech, Inc. E. Chang and M. Broder are employees of Partnership for Health Analytic Research, LLC, a health services research company paid by Genentech to conduct this research.

\section{Availability of data and materials}

The Nationwide Inpatient Sample (NIS) inpatient data that support the findings of this study are available for purchase from the Healthcare Cost and Utilization Project (HCUP).

\section{Authors' contributions}

All authors meet the ICMJE criteria for authorship. All authors were equally involved in the design of the study. EC conducted the statistical analyses and all authors contributed equally in the interpretation of results and writing of the manuscript. All authors read and approved the final manuscript.

\section{Competing interests}

K. Raimundo is an employee of Genentech, Inc. E. Chang and M. Broder are employees of Partnership for Health Analytic Research, LLC, a health services research company paid by Genentech to conduct this research.

\section{Consent for publication}

Not applicable.

\section{Ethics approval and consent to participate} Not applicable.

\section{Publisher's Note}

Springer Nature remains neutral with regard to jurisdictional claims in published maps and institutional affiliations.

\section{Author details}

${ }^{1}$ Stanford University, Stanford, CA, USA. ${ }^{2}$ Genentech, Inc, South San Francisco, CA, USA. ${ }^{3}$ Partnership for Health Analytic Research, LLC, Beverly Hills, CA, USA.
Received: 11 January 2017 Accepted: 11 May 2017

Published online: 22 May 2017

\section{References}

1. Raghu G, Chen S-Y, Yeh W-S, Maroni B, Li Q, Lee Y-C, et al. Idiopathic pulmonary fibrosis in US Medicare beneficiaries aged 65 years and older: incidence, prevalence, and survival, 2001-11. Lancet Respir Med. 2014;2:566-72.

2. Ryu JH, Moua T, Daniels CE, Hartman TE, Yi ES, Utz JP, et al. Idiopathic Pulmonary Fibrosis: Evolving Concepts. Mayo Clin Proc. 2014;89:1130-42.

3. Ley B, Collard HR, King TE. Clinical Course and Prediction of Survival in Idiopathic Pulmonary Fibrosis. Am J Respir Crit Care Med. 2011;183:431-40.

4. Martinez FJ, Safrin S, Weycker D, et al. The clinical course of patients with idiopathic pulmonary fibrosis. Ann Intern Med. 2005;142:963-7.

5. Song JW, Hong S-B, Lim C-M, et al. Acute exacerbation of idiopathic pulmonary fibrosis: incidence, risk factors and outcome. Eur Respir J. 2011;37:356-63.

6. Raghu G, Weycker D, Edelsberg J, et al. Incidence and Prevalence of Idiopathic Pulmonary Fibrosis. Am J Respir Crit Care Med. 2006;174:810-6.

7. King TE, Bradford WZ, Castro-Bernardini S, et al. A Phase 3 Trial of Pirfenidone in Patients with Idiopathic Pulmonary Fibrosis. N Engl J Med. 2014;370:2083-92.

8. Richeldi L, du Bois RM, Raghu G, et al. Efficacy and Safety of Nintedanib in Idiopathic Pulmonary Fibrosis. N Engl J Med. 2014;370:2071-82.

9. Weill D, Benden C, Corris PA, et al. A consensus document for the selection of lung transplant candidates: 2014-An update from the Pulmonary Transplantation Council of the International Society for Heart and Lung Transplantation. J Heart Lung Transplant. 2015;34:1-15.

10. Raghu G. Idiopathic pulmonary fibrosis: guidelines for diagnosis and clinical management have advanced from consensus-based in 2000 to evidencebased in 2011. Eur Respir J. 2011;37:743-6.

11. Simon-Blancal $V$, Freynet $\mathrm{O}$, Nunes $\mathrm{H}$, et al. Acute Exacerbation of Idiopathic Pulmonary Fibrosis: Outcome and Prognostic Factors. Respiration. 2012;83:28-35.

12. Vianello A, Arcaro G, Battistella L, et al. Noninvasive ventilation in the event of acute respiratory failure in patients with idiopathic pulmonary fibrosis. J Crit Care. 2014;29:562-7.

13. Blivet S, Philit F, Sab JM, et al. Outcome of patients with idiopathic pulmonary fibrosis admitted to the ICU for respiratory failure. Chest. 2001; 120:209-12.

14. Stern JB, Mal H, Groussard O, et al. Prognosis of patients with advanced idiopathic pulmonary fibrosis requiring mechanical ventilation for acute respiratory failure. Chest. 2001;120:213-9.

15. Fumeaux $T$, Rothmeier $C$, Jolliet $P$. Outcome of mechanical ventilation for acute respiratory failure in patients with pulmonary fibrosis. Intensive Care Med. 2001;27:1868-74.

16. Saydain G, Islam A, Afessa B, et al. Outcome of Patients with Idiopathic Pulmonary Fibrosis Admitted to the Intensive Care Unit. Am J Respir Crit Care Med. 2002;166:839-42.

17. Mallick S. Outcome of patients with idiopathic pulmonary fibrosis (IPF) ventilated in intensive care unit. Respir Med. 2008;102:1355-9.

18. Houchens R, Ross D, Elixhauser A, et al. Nationwide Inpatient Sample (NIS) Redesign Final Report. U.S. Agency for Healthcare Research and Quality. 2014 Apr. Report No.: 2014-04. http://www.hcup-us.ahrq.gov/reports/ methods/methods.jsp

19. APR DRG Software. 3 M Health Information Systems - US. http://solutions. $3 \mathrm{~m} . c o m / w p s / p o r t a l / 3 M / e n$ US/Health-Information-Systems/HIS/Productsand-Services/Products-List-A-Z/APR-DRG-Software/. Accessed 25 April 2016.

20. Hwang W, Weller W, Ireys $\mathrm{H}$, et al. Out-of-pocket medical spending for care of chronic conditions. Health Aff (Millwood). 2001;20:267-78.

21. Inflation Calculator. Bureau of Labor Statistics. http://www.bls.gov/data/ inflation_calculator.htm. Accessed 25 April 2016.

22. Baron RM, Kenny DA. The moderator-mediator variable distinction in social psychological research: conceptual, strategic, and statistical considerations. J Pers Soc Psychol. 1986:51:1173-82.

23. Collard HR, Ward AJ, Lanes $\mathrm{S}$, et al. Burden of illness in idiopathic pulmonary fibrosis. J Med Econ. 2012;15:829-35.

24. Collard HR, Chen S-Y, Yeh W-S, et al. Health care utilization and costs of idiopathic pulmonary fibrosis in U.S. Medicare beneficiaries aged 65 years and older. Ann Am Thorac Soc. 2015;12:981-7.

25. Raimundo K, Chang E, Broder MS, et al. Clinical and economic burden of idiopathic pulmonary fibrosis: a retrospective cohort study. BMC Pulm. Med. 2016. http://www.biomedcentral.com/1471-2466/16/2. Accessed 22 Jan 2016 
26. Wu N, Yu YF, Chuang C-C, et al. Healthcare resource utilization among patients diagnosed with idiopathic pulmonary fibrosis in the United States. J Med Econ. 2015;18:249-57.

27. Yu YF, Wu N, Chuang C-C, et al. Patterns and Economic Burden of Hospitalizations and Exacerbations Among Patients Diagnosed with Idiopathic Pulmonary Fibrosis. J Manag Care Spec Pharm. 2016;22:414-23.

28. Azuma A, Nukiwa T, Tsuboi E, et al. Double-blind, Placebo-controlled Trial of Pirfenidone in Patients with Idiopathic Pulmonary Fibrosis. Am J Respir Crit Care Med. 2005;171:1040-7.

29. Esposito DB, Lanes S, Donneyong $M$, et al. Idiopathic Pulmonary Fibrosis in United States Automated Claims. Incidence, Prevalence, and Algorithm Validation. Am J Respir Crit Care Med. 2015;192:1200-7.

30. Quan H, Li B, Duncan Saunders L, et al. Assessing Validity of ICD-9-CM and ICD-10 Administrative Data in Recording Clinical Conditions in a Unique Dually Coded Database: Assessing Validity of ICD-9-CM and ICD-10. Health Serv Res. 2008;43:1424-41.

Submit your next manuscript to BioMed Central and we will help you at every step:

- We accept pre-submission inquiries

- Our selector tool helps you to find the most relevant journal

- We provide round the clock customer support

- Convenient online submission

- Thorough peer review

- Inclusion in PubMed and all major indexing services

- Maximum visibility for your research

Submit your manuscript at www.biomedcentral.com/submit
Biomed Central 